\title{
Adaptive Tracking Control of a Wheeled Mobile Robot via an Uncalibrated Camera System
}

\author{
Warren E. Dixon, Member, IEEE, Darren M. Dawson, Senior Member, IEEE, Erkan Zergeroglu, Member, IEEE, \\ and Aman Behal
}

\begin{abstract}
This paper considers the problem of position/orientation tracking control of wheeled mobile robots via visual servoing in the presence of parametric uncertainty associated with the mechanical dynamics and the camera system. Specifically, we design an adaptive controller that compensates for uncertain camera and mechanical parameters and ensures global asymptotic position/orientation tracking. Simulation and experimental results are included to illustrate the performance of the control law.
\end{abstract}

Index Terms-Adaptive control, visual-servoing, wheeled mobile robot.

\section{INTRODUCTION}

A $\mathrm{S}$ the demand increases for wheeled mobile robots (WMRs) in settings that range from shopping centers, hospitals, warehouses, and nuclear waste facilities, the need for precise control of WMRs is clearly evident; hence, a closed-loop sensor-based controller is required. Unfortunately, due to the nonholonomic nature of the WMR and the standard encoder hardware configuration (e.g., optical encoders mounted on the actuators), the WMR Cartesian position is difficult to accurately obtain. That is, the linear velocity of the WMR must first be numerically differentiated from the position (i.e., by the backward difference algorithm) and then the nonlinear kinematic model must be numerically integrated to obtain the WMR Cartesian position. Since numerical differentiation/integration errors may accumulate over time, the accuracy of the numerically calculated WMR Cartesian position may be compromised. An interesting approach to overcome this position measurement problem is to utilize a vision system to directly obtain the Cartesian position information required by the controller (for an overview of the state-of-the-art in robot visual servoing, see [7] and [18]). Specifically, a ceiling-mounted

Manuscript received March 12, 2000; revised January 9, 2001. This work was supported in part by a Eugene P. Wigner Fellow and Staff Member at the Oak Ridge National Laboratory, managed by UT-Battelle, LLC, for the U.S Department of Energy under Contract DE-AC05-00OR22725. Additional support is provided by the U.S. National Science Foundation Grants DMI-9457967, CMS-9634796, ECS-9619785, DMI-9813213, and EPS-9630167, DOE Grant DE-FG07-96ER14728, a DOC Grant, and the Gebze Institute for Advanced Technology. This paper was recommended by Associate Editor R. A. Hess.

W. E. Dixon is with the Robotics and Process Systems Division, Oak Ridge National Laboratory, Oak Ridge, TN 37831 USA (e-mail: dixonwe@ornl.gov).

D. M. Dawson and A. Behal are with the Department of Electrical and Computer Engineering, Clemson University, Clemson, SC 29634 USA.

E. Zergeroglu was with the Department of Electrical and Computer Engineering, Clemson University, Clemson, SC 29634 USA. He is now with Optical Fiber Solutions, Bell Laboratory Innovations, Lucent Technologies, Sturbridge. MA 01566 USA.

Publisher Item Identifier S 1083-4419(01)05220-7. camera system can be used to determine the WMR Cartesian position without requiring numerical calculations. However, as emphasized by Bishop et al. in [1], when a vision system is utilized to extract information about a robot and the environment, adequate calibration of the vision system is required. That is, parametric uncertainty associated with the calibration of the camera corrupts the WMR position/orientation information; hence, camera calibration errors can result in degraded control performance.

Despite the above motivation to incorporate visual information in the control loop, most of the WMR research available in literature which incorporates visual information in the overall system seems to be concerned with vision-based navigation (i.e., using visual information for trajectory planning). It also seems that the state-of-the-art WMR research that specifically targets incorporating visual information from an on-board camera into the closed-loop control strategy can be found in [5], [15], [21]. Specifically, in [15], Ma et al. incorporates the dynamics of image curves obtained from a mobile camera system in the design of stabilizing control laws for tracking piecewise analytic curves. In [1], Espiau et al. proposed a visual servoing framework and in [5], Samson et al. address control issues in the image plane. For the most part, it seems that previous visual-servoing WMR work has assumed that the parametric uncertainty associated with the camera system can been neglected. In contrast, it seems that visual servoing research for robot manipulators has focused on the design of controllers that account for uncalibrated camera effects as well as uncertainty associated with the mechanical dynamics. Specifically, in [10], Kelly designed a setpoint controller to take into account uncertainties in the camera orientation to achieve a local asymptotically stable result; however, the controller required exact knowledge of the robot gravitational term and restricted the difference between the estimated and actual camera orientation to the interval $\left(-90^{\circ}, 90^{\circ}\right)$. In [1], Bishop and Spong developed an inverse dynamics-type, position tracking control scheme (i.e., exact model knowledge of the mechanical dynamics) with on-line adaptive camera calibration that guaranteed global asymptotic position tracking; however, convergence of the position tracking error required the desired position trajectory to be persistently exciting. In [16], Maruyama and Fujita proposed setpoint controllers for the camera-in-hand configuration; however, the proposed controllers required exact knowledge of the camera orientation and assumed the camera scaling factors to be the same value for both directions. In [11], Kelly et al. utilized a composite velocity inner loop, image-based outer loop fixed-camera 
tracking controller to obtain a local asymptotic stability result; however, exact model knowledge of the robot dynamics and a calibrated camera are required, and the difference between the estimated and actual camera orientation is restricted to the interval $\left(-90^{\circ}, 90^{\circ}\right)$. Recently, in [23], Zergeroglu et al. designed an adaptive tracking controller that accounted for parametric uncertainty throughout the entire robot-camera system; however, the controller required that the difference between the estimated and actual camera orientation be restricted to the interval $\left(-90^{\circ}, 90^{\circ}\right)$. Moreover, in [24], Zergeroglu et al. proposed a globally uniformly ultimately bounded (GUUB) tracking controller that is robust to uncertainty throughout the entire robot-camera system for a fixed-camera configuration, and a GUUB regulating controller for a camera-in-hand configuration. Note that in order to achieve the above results, [24] require that the camera orientation be within a certain range.

In this paper, we design a global asymptotic position/orientation tracking controller for a WMR with a ceiling-mounted fixed camera that adapts for uncertainty associated with the camera calibration (e.g., magnification factors, focal length, and orientation) in addition to the uncertainty associated with the mechanical parameters of the WMR dynamic model (e.g., mass, inertia, friction). We note that most of the vision-based navigation approaches found in WMR literature can be utilized to generate the camera-space reference trajectory for use in the proposed controller. However, if the camera is not assumed to be perfectly calibrated, then it is not obvious how to generate the reference trajectory in the task-space using the camera-system; hence, it seems that the reference trajectory must be generated in the camera-space and the control loop must be closed in the camera-space. Following this line of reasoning, we use a camera-space reference trajectory generator and the cameraspace WMR kinematic model to formulate an open-loop error system. This open-loop error system and the previous control structure given in [3] and [20] are then used to develop a kinematic control to ensure tracking in the camera-space. We then use the standard pin-hole camera model and the WMR cameraspace model to develop a transformation between the actual WMR velocity and the camera-space WMR velocity. This transformation is then used to transform the WMR dynamic model into a form that facilitates the design of a torque input adaptive controller that compensates for parametric uncertainty associated with camera calibration effects as well as the WMR mechanical dynamics. The proposed adaptive controller achieves global asymptotic tracking and requires the following signals for implementation:

1) WMR position/orientation in the camera-space;

2) WMR linear and angular velocity in the camera-space;

3) actual WMR orientation and angular velocity.

Note that the orientation and angular velocity of the WMR can be obtained from the on-board optical encoders and the backward difference algorithm while the WMR linear and angular velocity in the camera-space can be calculated from the WMR position/orientation in the camera-space using the backward difference algorithm; hence, the proposed controller does not require integration of the nonlinear kinematic model for obtaining the WMR Cartesian position.
This paper is organized as follows. In Section II, we describe the kinematic model of a WMR in the task-space and the camera-space, and then we utilize the pin-hole camera model to formulate a global invertible transformation between the two spaces. In Section III, the control objective of the paper is stated and then a kinematic tracking controller along with the corresponding open-loop error system is developed. In Section IV, we develop the dynamic model for the WMR that facilitates the subsequent closed-loop control development given in Section $\mathrm{V}$ and the corresponding stability analysis given in Section VI. In Section VII, the controller's performance is illustrated through simulation and experimental results. In Section VIII, we present some concluding remarks.

\section{Kinematic Model}

\section{A. WMR Kinematic Model in the Task-Space}

The kinematic model of a WMR in the task-space is assumed to be of the following form [17]:

$$
\dot{q}=S(q) v
$$

where $q(t), \dot{q}(t) \in \Re^{3}$ are defined as

$$
q=\left[\begin{array}{lll}
x_{c} & y_{c} & \theta
\end{array}\right]^{T} \quad \dot{q}=\left[\begin{array}{lll}
\dot{x}_{c} & \dot{y}_{c} & \dot{\theta}
\end{array}\right]^{T}
$$

$x_{c}(t), y_{c}(t)$, and $\theta(t) \in \Re^{1}$ denote the position and orientation, respectively, of the center of mass (COM) of the WMR (which is assumed to coincide with the center of the axis of rotation of the WMR), $\dot{x}_{c}(t), \dot{y}_{c}(t)$ denote the Cartesian components of the linear velocity of the COM, $\dot{\theta}(t) \in \Re^{1}$ denotes the angular velocity of the COM, the matrix $S(q) \in \Re^{3 \times 2}$ is defined as follows:

$$
S(q)=\left[\begin{array}{cc}
\cos \theta & 0 \\
\sin \theta & 0 \\
0 & 1
\end{array}\right]
$$

and the velocity vector $v(t) \in \Re^{2}$ is defined as

$$
v=\left[\begin{array}{ll}
v_{1} & v_{2}
\end{array}\right]^{T}=\left[\begin{array}{ll}
v_{l} & \dot{\theta}
\end{array}\right]^{T}
$$

with $v_{l}(t) \in \Re^{1}$ denoting the linear velocity of the COM of the WMR.

\section{B. WMR Kinematic Model in the Camera-Space}

Based on the task-space kinematic formulation given in (1) and the desire to craft a camera-space tracking controller, we assume that the representation of the WMR kinematic model in the camera-space takes the following form

$$
\dot{\bar{q}}=S(\bar{q}) \bar{v}
$$

where $S(\cdot)$ was defined in $(3), \bar{q}(t)=\left[\bar{x}_{c}(t) \bar{y}_{c}(t) \bar{\theta}(t)\right]^{T} \in \Re^{3}$ denotes the position and orientation of the WMR in the cameraspace, and $\bar{v}(t)=\left[\bar{v}_{1}(t) \bar{v}_{2}(t)\right]^{T} \in \Re^{2}$ denotes the linear and angular velocity of the WMR in the camera-space. That is, we assume that the WMR in the camera-space must satisfy the same kinematic model as the WMR in the task-space. With regard to the robot-camera system configuration, it is assumed that the 
camera is fixed above the robot workspace such that we have the following.

1) Its image plane is parallel to the plane of motion of the robot.

2) The camera can capture images throughout the entire robot workspace.

3) The camera system can determine the COM of the WMR by recognizing some physical characteristic (e.g., a light emitting diode).

4) The camera can determine the orientation of the WMR, and hence, the direction that the WMR is traveling, by recognizing an additional characteristic (e.g., an arrow painted on the WMR, a second light emitting diode, etc).

\section{Task-Space to Camera-Space Transformations}

In order to force the WMR to track the reference trajectory in the camera-space, we are motivated to relate the kinematic control inputs [i.e., $\bar{v}(t)]$ in the camera-space to the kinematic control inputs [i.e., $v(t)$ ] in the task-space. To this end, we utilize the so-called pin-hole lens model [1] for the robot-camera system to express the WMR camera-space position vector in terms of the task-space position vector as shown below (see Fig. 1)

$$
\left[\begin{array}{l}
\bar{x}_{c}(t) \\
\bar{y}_{c}(t)
\end{array}\right]=H R\left(\theta_{0}\right)\left(\left[\begin{array}{l}
x_{c}(t) \\
y_{c}(t)
\end{array}\right]-\left[\begin{array}{l}
O_{o 1} \\
O_{o 2}
\end{array}\right]\right)+\left[\begin{array}{l}
O_{i 1} \\
O_{i 2}
\end{array}\right]
$$

where $H \in \Re^{2 \times 2}$ is a diagonal, positive-definite, constant matrix defined as follows:

$$
H=\left[\begin{array}{cc}
\alpha_{1} & 0 \\
0 & \alpha_{2}
\end{array}\right]
$$

$\alpha_{1}, \alpha_{2} \in \Re^{1}$ are positive constants defined as follows:

$$
\alpha_{1}=\beta_{1} \frac{\lambda}{z} \quad \alpha_{2}=\beta_{2} \frac{\lambda}{z}
$$

$z \in \Re^{1}$ represents the constant height of the camera's optical center with respect to the task-space plane, $\lambda \in \Re^{1}$ is a constant representing the camera's focal length, the positive constants denoted by $\beta_{1}, \beta_{2} \in \Re^{1}$ represent the camera's constant scale factors (in pixels $/ \mathrm{m}$ ) along their respective Cartesian directions, respectively, $R\left(\theta_{0}\right) \in \Re^{2 \times 2}$ is a constant, rotation matrix defined by

$$
R\left(\theta_{0}\right)=\left[\begin{array}{cc}
\cos \left(\theta_{0}\right) & -\sin \left(\theta_{0}\right) \\
\sin \left(\theta_{0}\right) & \cos \left(\theta_{0}\right)
\end{array}\right]
$$

$\theta_{0}$ represents the constant, clockwise rotation angle of the camera coordinate system with respect to the task-space coordinate system, $\left[O_{o 1}, O_{o 2}\right]^{T} \in \Re^{2}$ denotes a projection of the camera's optical center on the task-space plane, and $\left[O_{i 1}, O_{i 2}\right]^{T} \in \Re^{2}$ denotes the image center which is defined as the frame buffer coordinates of the intersection of the optical axis with the image plane (see [13] for explicit details).

To relate $\bar{v}_{1}(t)$ to $v_{1}(t)$, we first take the time derivative of (6) and substitute (1) for the time derivative of (2) to obtain the following relationship:

$$
\left[\begin{array}{c}
\dot{\bar{x}}_{c} \\
\dot{\bar{y}}_{c}
\end{array}\right]=\left[\begin{array}{c}
v_{1} \alpha_{1} \cos \left(\theta+\theta_{0}\right) \\
v_{1} \alpha_{2} \sin \left(\theta+\theta_{0}\right)
\end{array}\right] \text {. }
$$

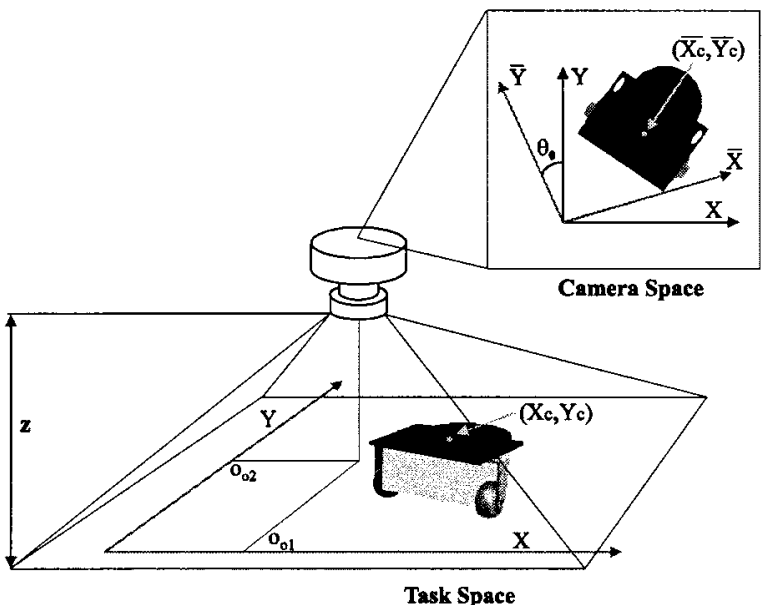

Fig. 1. Robot-camera system configuration.

After multiplying $\dot{\bar{x}}_{c}(t)$ of (10) by $\left(1 / \alpha_{1}\right) \cos \left(\theta+\theta_{0}\right)$, multiplying $\dot{\bar{y}}_{c}(t)$ of (10) by $\left(1 / \alpha_{2}\right) \sin \left(\theta+\theta_{0}\right)$, and substituting (5) for $\dot{\bar{x}}_{c}(t)$ and $\dot{\bar{y}}_{c}(t)$, we obtain the following expression:

$$
\left[\begin{array}{c}
\frac{1}{\alpha_{1}} \bar{v}_{1} \cos (\bar{\theta}) \cos \left(\theta+\theta_{0}\right) \\
\frac{1}{\alpha_{2}} \bar{v}_{1} \sin (\bar{\theta}) \sin \left(\theta+\theta_{0}\right)
\end{array}\right]=\left[\begin{array}{l}
v_{1} \cos ^{2}\left(\theta+\theta_{0}\right) \\
v_{1} \sin ^{2}\left(\theta+\theta_{0}\right)
\end{array}\right] .
$$

After adding rows of the vector equality given by (11), we have

$$
v_{1}=T_{1} \bar{v}_{1}
$$

where $T_{1}(\theta(t), \bar{\theta}(t)) \in \Re^{1}$ is a positive scalar function defined as

$$
\begin{aligned}
T_{1}= & \frac{1}{\alpha_{1}} \cos (\bar{\theta}) \cos \left(\theta+\theta_{0}\right) \\
& +\frac{1}{\alpha_{2}} \sin (\bar{\theta}) \sin \left(\theta+\theta_{0}\right)>\gamma_{1} .
\end{aligned}
$$

$\alpha_{1}$ and $\alpha_{2}$ were defined in (8), and $\gamma_{1} \in \Re^{1}$ is some positive constant (see Appendix A for explicit details).

To relate $\bar{v}_{2}(t)$ to $v_{2}(t)$, we eliminate $\bar{v}_{1}(t)$ in the first two rows of the vector equality given by (5) to conclude that

$$
\dot{\bar{y}}_{c}=\dot{\bar{x}}_{c} \tan \bar{\theta}
$$

hence, after substituting (10) for $\dot{\bar{y}}_{c}(t)$ and $\dot{\bar{x}}_{c}(t)$ into (14), we obtain the following relationship:

$$
\tan \left(\theta+\theta_{0}\right)=\frac{\alpha_{1}}{\alpha_{2}} \tan \bar{\theta}
$$

After taking the time derivative of (15) and then performing some algebraic manipulation, we have that

$$
\dot{\theta}=T_{2} \dot{\bar{\theta}}
$$

where $T_{2}(\theta(t)) \in \Re^{1}$ is a positive scalar function defined as

$$
T_{2}=\frac{\alpha_{1}}{\alpha_{2}} \cos ^{2}\left(\theta+\theta_{0}\right)+\frac{\alpha_{2}}{\alpha_{1}} \sin ^{2}\left(\theta+\theta_{0}\right)>\gamma_{2}
$$

where $\alpha_{1}$ and $\alpha_{2}$ were defined in (8), and $\gamma_{2} \in \Re^{1}$ is some positive constant. Based on (12)) and (16), we can now formulate a 
global invertible transformation between the task-space WMR velocities and the camera-space WMR velocities as follows:

$$
v=T_{0} \bar{v}
$$

where $T_{0}(\theta(t), \bar{\theta}(t)) \in \Re^{2 \times 2}$ is defined as

$$
T_{0}=\left[\begin{array}{cc}
T_{1} & 0 \\
0 & T_{2}
\end{array}\right]
$$

and the positive scalar functions $T_{1}(\theta(t), \bar{\theta}(t))$, and $T_{2}(\theta(t))$ were defined in (13) and (17), respectively.

\section{KINEMATIC CONTROL FORMULATION}

\section{A. Control Objective}

The primary control objective is to force the representation of the WMR in the camera-space to track a trajectory generated in the camera-space in the presence of parametric uncertainty (i.e., the camera calibration parameters and the mechanical parameters associated with the dynamic model). Similar to previous WMR research performed in the task-space (e.g., see [8] and [9]), the desired trajectory of the WMR is generated via a reference robot which moves in the camera-space according to the following dynamic trajectory:

$$
\dot{\bar{q}}_{r}=S\left(\bar{q}_{r}\right) \bar{v}_{r}
$$

where $S(\cdot)$ was defined in $(3), \bar{q}_{r}(t)=\left[\bar{x}_{c r}(t) \bar{y}_{c r}(t) \bar{\theta}_{r}(t)\right]^{T}$ $\in \Re^{3}$ denotes the reference position and orientation trajectory in the camera-space, and $\bar{v}_{r}(t)=\left[\bar{v}_{r 1}(t) \bar{v}_{r 2}(t)\right]^{T} \in \Re^{2} \mathrm{de}-$ notes the reference linear and angular velocity of the WMR in the camera-space. With regard to (20), it is assumed that the signal $\bar{v}_{r}(t)$ is obtained from a path planning algorithm and is constructed to produce the desired motion in the camera-space and that $\bar{v}_{r}(t), \dot{\bar{v}}_{r}(t), \bar{q}_{r}(t)$, and $\dot{\bar{q}}_{r}(t)$ are bounded for all time.

\section{B. Open-Loop Error System Development}

To facilitate the subsequent closed-loop error system development and stability analysis, we define an auxiliary error signal denoted by $e(t)=\left[\begin{array}{lll}e_{1}(t) & e_{2}(t) & e_{3}(t)\end{array}\right]^{T} \in \Re^{3}$, that is related to the difference between the reference position/orientation and the camera-space position/orientation of the WMR through a global invertible transformation as follows [3], [20]:

$$
\left[\begin{array}{l}
e_{1} \\
e_{2} \\
e_{3}
\end{array}\right]=\left[\begin{array}{ccc}
\cos (\bar{\theta}) & \sin (\bar{\theta}) & 0 \\
-\sin (\bar{\theta}) & \cos (\bar{\theta}) & 0 \\
0 & 0 & 1
\end{array}\right]\left[\begin{array}{l}
\tilde{x} \\
\tilde{y} \\
\tilde{\theta}
\end{array}\right]
$$

where $\tilde{x}(t), \tilde{y}(t) \in \Re^{1}$, and $\tilde{\theta}(t) \in \Re^{1}$ are defined as

$$
\tilde{x}=\bar{x}_{c r}-\bar{x}_{c} \quad \tilde{y}=\bar{y}_{c r}-\bar{y}_{c} \quad \tilde{\theta}=\bar{\theta}_{r}-\bar{\theta} .
$$

We can now formulate the open-loop error dynamics for $e(t)$ by differentiating (21) to obtain the following expression:

$$
\left[\begin{array}{c}
\dot{e}_{1} \\
\dot{e}_{2} \\
\dot{e}_{3}
\end{array}\right]=\left[\begin{array}{c}
\bar{v}_{2} e_{2}+\bar{u}_{1} \\
-\bar{v}_{2} e_{1}+\bar{v}_{r 1} \sin e_{3} \\
\bar{u}_{2}
\end{array}\right]
$$

where $\bar{u}(t)=\left[\bar{u}_{1}(t) \bar{u}_{2}(t)\right]^{T} \in \Re^{2}$ is an auxiliary signal defined in terms of the camera-space orientation/velocity, and the desired trajectory as follows:

$$
\bar{u}=-\bar{v}+\Pi
$$

with the auxiliary variable $\Pi\left(\bar{\theta}(t), \bar{\theta}_{r}(t), \bar{v}_{r}(t)\right) \in \Re^{2}$ being defined as follows:

$$
\Pi=\left[\begin{array}{c}
\bar{v}_{r 1} \cos e_{3} \\
\bar{v}_{r 2}
\end{array}\right] .
$$

To facilitate the kinematic closed-loop error system development, we inject the auxiliary control inputs, denoted by $\bar{u}_{d}(t)=$ $\left[\begin{array}{ll}\bar{u}_{d 1} & \bar{u}_{d 2}\end{array}\right] \in \Re^{2}$, into the open-loop dynamics of $e_{1}(t)$ and $e_{3}(t)$ by adding and subtracting $\bar{u}_{d 1}(t)$ and $\bar{u}_{d 2}(t)$ to the right-side of (23) to obtain the following expression:

$$
\left[\begin{array}{c}
\dot{e}_{1} \\
\dot{e}_{2} \\
\dot{e}_{3}
\end{array}\right]=\left[\begin{array}{c}
\bar{v}_{2} e_{2}+\bar{u}_{d 1}-\eta_{1} \\
-\bar{v}_{2} e_{1}+\bar{v}_{r 1} \sin e_{3} \\
\bar{u}_{d 2}-\eta_{2}
\end{array}\right]
$$

where the kinematic tracking error signal, denoted by $\eta(t) \epsilon$ $\Re^{2}$, is defined as follows:

$$
\eta=\left[\begin{array}{ll}
\eta_{1} & \eta_{2}
\end{array}\right]=\bar{u}_{d}-\bar{u}
$$

\section{Control Design and Closed-Loop Error System Development}

Based on (26) and the subsequent closed-loop error system development, we design $\bar{u}_{d}(t)$ as shown below [3], [20]

$$
\left[\begin{array}{l}
\bar{u}_{d 1} \\
\bar{u}_{d 2}
\end{array}\right]=\left[\begin{array}{c}
-k_{1} e_{1} \\
-\frac{\bar{v}_{r 1} \sin \left(e_{3}\right) e_{2}}{e_{3}}-k_{3} e_{3}
\end{array}\right]
$$

where $k_{1}, k_{3} \in \Re^{1}$ denote positive constant control gains. After substituting (28) into (26), the resulting kinematic closed-loop error system for $e(t)$ is given as follows:

$$
\left[\begin{array}{c}
\dot{e}_{1} \\
\dot{e}_{2} \\
\dot{e}_{3}
\end{array}\right]=\left[\begin{array}{c}
-k_{1} e_{1}+\bar{v}_{2} e_{2}-\eta_{1} \\
-\bar{v}_{2} e_{1}+\bar{v}_{r 1} \sin e_{3} \\
-k_{3} e_{3}-\frac{\bar{v}_{r 1} \sin \left(e_{3}\right) e_{2}}{e_{3}}-\eta_{2}
\end{array}\right] .
$$

\section{WMR DYNAMIC MODEL}

Since the proposed control is designed to include the effects of the dynamic model, we will assume that the task-space dynamic model for the WMR can be expressed as follows:

$$
M \dot{v}+F(v)=B \tau
$$

where $\dot{v}(t) \in \Re^{2}$ denotes the time derivative of $v(t)$ defined in (4), $M \in \Re^{2 \times 2}$ represents the constant, diagonal inertia matrix, $F(v) \in \Re^{2}$ represents the friction effects, $\tau(t) \in \Re^{2}$ represents the torque input vector, and $B \in \Re^{2 \times 2}$ represents a diagonal input matrix that governs torque transmission. To facilitate the subsequent control design, we transform the dynamic model into a form that is consistent with the kinematic transformation given by (18) and (24). Specifically, we premultiply (30) by $-T_{0}^{T}$, substitute (18) for $v(t)$, and then substitute (24) 
for $\bar{v}(t)$ in the resulting expression to obtain the following convenient dynamic model:

$$
\bar{M} \dot{\bar{u}}+\bar{V}_{m} \bar{u}+\bar{N}=\bar{B} \tau
$$

where

$$
\begin{aligned}
\bar{M}(\bullet) & =T_{0}^{T} M T_{0} \\
\bar{V}_{m}(\bullet) & =T_{0}^{T} M \dot{T}_{0} \bar{N}(\bullet) \\
\left.\bar{v}_{r}(t), \dot{\bar{v}}_{r}(t), \bar{u}(t)\right) & =-T_{0}^{T}\left(M\left(\dot{T}_{0} \Pi+T_{0} \dot{\Pi}\right)+F\left(T_{0}(-\bar{u}+\Pi)\right)\right)
\end{aligned}
$$

and $\bar{B}(\theta(t), \bar{\theta}(t))=-T_{0}^{T} B$. To facilitate the stability analysis, we note that the dynamic model given by (31) exhibits the following properties [14].

Property 1: The transformed inertia matrix $\bar{M}(\bullet)$ is symmetric, positive definite, and satisfies the following inequalities:

$$
m_{1}\|\xi\|^{2} \leq \xi^{T} \bar{M} \xi \leq m_{2}\|\xi\|^{2} \quad \forall \xi \in \Re^{2}
$$

where $m_{1}$ and $m_{2}$ are known positive constants, and $\|\cdot\|$ denotes the standard Euclidean norm.

Property $2: \because$ A skew-symmetric relationship exists between the transformed inertia matrix and the auxiliary matrix $\bar{V}_{m}(\cdot)$ as follows:

$$
\xi^{T}\left(\frac{1}{2} \dot{\bar{M}}-\bar{V}_{m}\right) \xi=0 \quad \forall \xi \in \Re^{2}
$$

where $\dot{M}(\cdot)$ represents the time derivative of the transformed inertia matrix.

Property 3: The robot dynamics given in (31) can be linearly parameterized as follows:

$$
Y_{0} \vartheta_{0}=\bar{M} \dot{\bar{u}}+\bar{V}_{m} \bar{u}+\bar{N}
$$

where $\vartheta_{0} \in \Re^{p}$ contains the unknown constant mechanical parameters (i.e., inertia, mass, and friction effects) and calibration/camera constants (i.e., $\theta_{0}, \alpha_{1}$, and $\alpha_{2}$ ) and $Y_{0}(\bar{u}, \dot{\bar{u}}, t) \in$ $\Re^{2 \times p}$ denotes the known regression matrix. Furthermore, the global invertible matrix $T_{0}(\theta(t), \bar{\theta}(t))$ defined in (19) is linearly parameterizable as shown below in the following:

$$
T_{0}=\left[\begin{array}{cc}
T_{1} & 0 \\
0 & T_{2}
\end{array}\right]=\left[\begin{array}{cc}
y_{1} \vartheta_{1} & 0 \\
0 & y_{2} \vartheta_{2}
\end{array}\right]
$$

where $\vartheta_{1} \in \Re^{p_{1}}, \vartheta_{2} \in \Re^{p_{2}}$ contain the unknown camera calibration constants, and $y_{1}(\theta(t), \bar{\theta}(t)) \in \Re^{1 \times p_{1}}, y_{2}(\theta(t)) \in$ $\Re^{1 \times p_{2}}$ denote known regression vectors.

Property 4: To avoid singularities in the subsequent control law, we now define convex regions, in the same manner as [2] and [12], for the parameter vectors $\vartheta_{1}$ and $\vartheta_{2}$ defined in (35). Specifically, based on (13), (17), and (35), we define the space spanned by the vector functions $y_{1}(\theta(t), \bar{\theta}(t))$ and $y_{2}(\theta(t))$ as follows:

$$
\begin{aligned}
& Y_{1}=\left\{y_{1}: y_{1}=y_{1}(\theta(t), \bar{\theta}(t)), \forall \theta(t), \bar{\theta}(t) \in \Re^{1}\right\} \\
& Y_{2}=\left\{y_{2}: y_{2}=y_{2}(\theta(t)), \forall \theta(t) \in \Re^{1}\right\} .
\end{aligned}
$$

In addition, we define the regions $\Lambda_{1}$ and $\Lambda_{2}$ as

$$
\begin{aligned}
& \Lambda_{1}=\left\{s_{1}: y_{1} s_{1} \geq \gamma_{1}, \forall y_{1} \in Y_{1}\right\} \\
& \Lambda_{2}=\left\{s_{2}: y_{2} s_{2} \geq \gamma_{2}, \forall y_{2} \in Y_{2}\right\}
\end{aligned}
$$

where $\gamma_{1}, \gamma_{2}$ were defined in (13) and (17), respectively, and we have the following definitions concerning the regions $\Lambda_{1}$ and $\Lambda_{2}$ and the subsequently designed parameter estimate vectors $\hat{\vartheta}_{1}(t) \in \Re^{p 1}$ and $\hat{\vartheta}_{2}(t) \in \Re^{p 2}$ : int $\left(\Lambda_{i}\right)$ is the interior of the region $\Lambda_{i}, \partial\left(\Lambda_{i}\right)$ is the boundary for the region $\Lambda_{i}, \hat{\vartheta}_{i}^{\perp} \in \Re^{p i}$ is a unit vector normal to $\partial\left(\Lambda_{i}\right)$ at the point of intersection of the boundary surface $\partial\left(\Lambda_{i}\right)$ and $\hat{\vartheta}_{i}$ where the positive direction for $\hat{\vartheta}_{i}^{\perp}$ is defined as pointing away from $\operatorname{int}\left(\Lambda_{i}\right)$ [note $\hat{\vartheta}_{i}^{\perp}$ is only defined for $\left.\hat{\vartheta}_{i} \in \partial\left(\Lambda_{i}\right)\right], P_{r}^{t}\left(\mu_{i}\right)$ is the component of the vector $\mu_{i} \in \Re^{p i}$ that is tangential to $\partial\left(\Lambda_{i}\right)$ at the point of intersection of the boundary surface $\partial\left(\Lambda_{i}\right)$ and the vector $\hat{\vartheta}_{i}, P_{r}^{\perp}\left(\mu_{i}\right)=$ $\mu_{i}-P_{r}^{t}\left(\mu_{i}\right) \in \Re^{p i}$ is the component of the vector $\mu_{i} \in \Re^{p i}$, that is perpendicular to $\partial\left(\Lambda_{i}\right)$ at the point of intersection of the boundary surface $\partial\left(\Lambda_{i}\right)$ and the vector $\hat{\vartheta}_{i}$ for $i=1,2$.

Remark 1: Note that the subsequent control development relies heavily on the fact that the dynamic model is decoupled. It is not obvious how the controller can be extended to account for additional coupling terms that result from the COM of the WMR not corresponding to the center of the axis of rotation. Future research will target the development of a controller that can track a desired camera-space trajectory despite the use of an uncalibrated vision system and parametric uncertainty in the WMR dynamic model which includes the additional coupling effects.

\section{DynAmic Model CONTROL Formulation}

We now utilize the dynamic model given by (31) to design a control torque input that regulates the kinematic tracking error signal defined in (22). Motivated by the structure of (29), the kinematic tracking control objective can be obtained by regulating the auxiliary tracking signal defined in (27). To this end, we develop the closed-loop error system for $\eta(t)$ by taking the time derivative of (27) and multiplying both sides of the resulting expression by $\bar{M}(\cdot)$ to obtain the following expression:

$$
\bar{M} \dot{\eta}=\bar{M} \dot{\bar{u}}_{d}-\bar{M} \dot{\bar{u}} .
$$

After substituting (31) for $\bar{M} \dot{\bar{u}}$ and then adding and subtracting terms to the right-side of the resulting expression, we arrange the dynamics in the following advantageous form

$$
\begin{aligned}
\bar{M} \dot{\eta}= & -\bar{V}_{m} \eta+Y_{d 0}\left(\vartheta_{0}-\hat{\vartheta}_{0}\right)-\bar{B} \tau \\
& +\left[\begin{array}{l}
-k_{\eta 1} \eta_{1}+e_{1} \\
-k_{\eta 2} \eta_{2}+e_{3}
\end{array}\right]+\left[\begin{array}{l}
Y_{d 01} \hat{\vartheta}_{0}+k_{\eta 1} \eta_{1}-e_{1} \\
Y_{d 02} \hat{\vartheta}_{0}+k_{\eta 2} \eta_{2}-e_{3}
\end{array}\right]
\end{aligned}
$$

where (27) was utilized, and regression matrix parametrization $Y_{d 0} \vartheta_{0}$ is defined according to

$$
Y_{d 0} \vartheta_{0}=\bar{M} \dot{\bar{u}}_{d}+\bar{V}_{m} \bar{u}_{d}+\bar{N}
$$

where $Y_{d 0}(\cdot) \in \Re^{2 \times p}$ denotes the known desired regression matrix, and $\vartheta_{0}$ was defined in (34). 
Based on the subsequent stability proof and the regulation of $\eta(t)$, we design the control torque input $\tau(t)$ as follows:

$$
\tau(t)=B^{-1} \tau_{0}
$$

where $\tau_{0}(t)=\left[\begin{array}{ll}\tau_{01} & \tau_{02}\end{array}\right] \in \Re^{2}$ is an auxiliary control signal designed as shown in the following:

$$
\left[\begin{array}{l}
\tau_{01} \\
\tau_{02}
\end{array}\right]=\left[\begin{array}{l}
-\left(\frac{1}{y_{1} \hat{\vartheta}_{1}}\right)\left(Y_{d 01} \hat{\vartheta}_{0}+k_{\eta 1} \eta_{1}-e_{1}\right) \\
-\left(\frac{1}{y_{2} \hat{\vartheta}_{2}}\right)\left(Y_{d 02} \hat{\vartheta}_{0}+k_{\eta 2} \eta_{2}-e_{3}\right)
\end{array}\right]
$$

where $k_{\eta 1}, k_{\eta 2} \in \Re^{1}$ are positive constant control gains, and the parameter update laws for $\hat{\vartheta}_{0}(t), \hat{\vartheta}_{1}(t)$, and $\hat{\vartheta}_{2}(t)$ are designed as follows:

$$
\begin{aligned}
& \dot{\hat{\vartheta}}_{0}=\Gamma_{0} Y_{d 0}^{T} \eta \\
& \dot{\hat{\vartheta}}_{i}= \begin{cases}\Omega_{i}, & \text { if } \hat{\vartheta}_{i} \in \operatorname{int}\left(\Lambda_{i}\right) \\
\Omega_{i}, & \text { if } \hat{\vartheta}_{i} \in \partial\left(\Lambda_{i}\right) \text { and } \Omega_{i}^{T} \hat{\vartheta}_{i}^{\perp} \leq 0 \\
P_{r}^{t}\left(\Omega_{i}\right), & \text { if } \hat{\vartheta}_{i} \in \partial\left(\Lambda_{i}\right) \text { and } \Omega_{i}^{T} \hat{\vartheta}_{i}^{\perp}>0\end{cases}
\end{aligned}
$$

where $\hat{\vartheta}_{i}(0) \in \operatorname{int}\left(\Lambda_{i}\right)$ for $i=1,2$, the auxiliary signals $\Omega_{1}(t) \in$ $\Re^{p 1}$ and $\Omega_{2}(t) \in \Re^{p 2}$ are defined as follows:

$$
\Omega_{1}=-\Gamma_{1} y_{1}^{T} \eta_{1} \frac{1}{y_{1} \hat{\vartheta}_{1}}\left(Y_{d 01} \hat{\vartheta}_{0}+k_{\eta 1} \eta_{1}-e_{1}\right)
$$

and

$$
\Omega_{2}=-\Gamma_{2} y_{2}^{T} \eta_{2} \frac{1}{y_{2} \hat{\vartheta}_{2}}\left(Y_{d 02} \hat{\vartheta}_{0}+k_{\eta 2} \eta_{2}-e_{3}\right)
$$

and $\Gamma_{0} \in \Re^{p \times p}, \Gamma_{1} \in \Re^{p 1 \times p 1}, \Gamma_{2} \in \Re^{p 2 \times p 2}$ are positive definite gain matrices. If $\hat{\vartheta}_{i}(0) \in \operatorname{int}\left(\Lambda_{i}\right)$, the above update law for $\hat{\vartheta}_{1}(t)$ and $\hat{\vartheta}_{2}(t)$ defined in (44) ensures that $y_{1} \hat{\vartheta}_{1}>\gamma_{1}$ and $y_{2} \hat{\vartheta}_{2}>\gamma_{2}$ (See the definitions given in Property 4 and the explanations given in [2] and [12]). After utilizing (35), (41), (42), and the definition of $\bar{B}(\theta(t), \bar{\theta}(t))$ given in (31) and then performing some algebraic manipulations, we can obtain the following expression for the closed-loop error system for $\eta(t)$ :

$$
\begin{aligned}
\bar{M} \dot{\eta}= & -\bar{V}_{m} \eta+Y_{d 0} \tilde{\vartheta}_{0}+\left[\begin{array}{l}
-k_{\eta 1} \eta_{1}+e_{1} \\
-k_{\eta 2} \eta_{2}+e_{3}
\end{array}\right] \\
& -\left[\begin{array}{l}
\left(\frac{1}{y_{1} \hat{\vartheta}_{1}}\right) y_{1} \tilde{\vartheta}_{1}\left(Y_{d 01} \hat{\vartheta}_{0}+k_{\eta 1} \eta_{1}-e_{1}\right) \\
\left(\frac{1}{y_{2} \hat{\vartheta}_{2}}\right) y_{2} \tilde{\vartheta}_{2}\left(Y_{d 02} \hat{\vartheta}_{0}+k_{\eta 2} \eta_{2}-e_{3}\right)
\end{array}\right]
\end{aligned}
$$

where the parameter error signals, denoted by $\tilde{\vartheta}_{0}(t) \in \Re^{p}$, $\tilde{\vartheta}_{1}(t) \in \Re^{p_{1}}, \tilde{\vartheta}_{2}(t) \in \Re^{p_{2}}$, are defined as follows:

$$
\tilde{\vartheta}_{0}=\vartheta_{0}-\hat{\vartheta}_{0}, \quad \tilde{\vartheta}_{1}=\vartheta_{1}-\hat{\vartheta}_{1}, \quad \tilde{\vartheta}_{2}=\vartheta_{2}-\hat{\vartheta}_{2} .
$$

\section{COMPOSITE STABILITY ANALYSIS}

Theorem 1: The control torque input given in (28), (41)-(46) along with the closed-loop error system given in (29) and (47) ensures global asymptotic position and orientation tracking control in the sense that

$$
\lim _{t \rightarrow \infty} \tilde{x}(t), \tilde{y}(t), \tilde{\theta}(t)=0
$$

provided

$$
\lim _{t \rightarrow \infty} \bar{v}_{r 1}(t) \neq 0
$$

where $\tilde{x}(t), \tilde{y}(t)$, and $\tilde{\theta}(t)$ are defined in (22).

Proof: To prove Theorem 1, we define a nonnegative, scalar function denoted by $V_{1}\left(e(t), \eta(t), \tilde{\vartheta}_{0}(t), \tilde{\vartheta}_{1}(t), \tilde{\vartheta}_{2}(t)\right)$ $\in \Re^{1}$ as follows:

$$
\begin{aligned}
V_{1}= & \frac{1}{2} e_{1}^{2}+\frac{1}{2} e_{2}^{2}+\frac{1}{2} e_{3}^{2}+\frac{1}{2} \eta^{T} \bar{M} \eta \\
& +\frac{1}{2} \tilde{\vartheta}_{0}^{T} \Gamma_{0}^{-1} \tilde{\vartheta}_{0}+\frac{1}{2} \tilde{\vartheta}_{1}^{T} \Gamma_{1}^{-1} \tilde{\vartheta}_{1}+\frac{1}{2} \tilde{\vartheta}_{2}^{T} \Gamma_{2}^{-1} \tilde{\vartheta}_{2} .
\end{aligned}
$$

After taking the time derivative of (51) and making the appropriate substitutions from (29) and (47), we can conclude that

$$
\begin{aligned}
\dot{V}_{1}= & e_{1}\left(\bar{v}_{2} e_{2}-k_{1} e_{1}-\eta_{1}\right)+e_{2}\left(-\bar{v}_{2} e_{1}+\bar{v}_{r 1} \sin e_{3}\right) \\
& +e_{3}\left(-\frac{\bar{v}_{r 1} \sin \left(e_{3}\right) e_{2}}{e_{3}}-k_{3} e_{3}-\eta_{2}\right) \\
& +\eta^{T}\left(Y_{d 0} \tilde{\vartheta}_{0}+\left[\begin{array}{l}
-k_{\eta 1} \eta_{1}+e_{1} \\
-k_{\eta 2} \eta_{2}+e_{3}
\end{array}\right]\right. \\
& \left.-\left[\left(\frac{1}{y_{1} \hat{\vartheta}_{1}}\right) y_{1} \tilde{\vartheta}_{1}\left(Y_{d 01} \hat{\vartheta}_{0}+k_{\eta 1} \eta_{1}-e_{1}\right)\right]\right) \\
& \left.-\left[\left(\frac{1}{y_{2} \hat{\vartheta}_{2}}\right) y_{2} \tilde{\vartheta}_{2}\left(Y_{d 02} \hat{\vartheta}_{0}+k_{\eta 2} \eta_{2}-e_{3}\right)\right]\right) \\
& -\tilde{\vartheta}_{0}^{T} \Gamma_{0}^{-1} \hat{\hat{\vartheta}}_{0}-\tilde{\vartheta}_{1}^{T} \Gamma_{1}^{-1} \dot{\hat{\vartheta}}_{1}-\tilde{\vartheta}_{2}^{T} \Gamma_{2}^{-1} \dot{\hat{\vartheta}}_{2}
\end{aligned}
$$

where (33) and the fact that

$$
\begin{aligned}
& \dot{\tilde{\vartheta}}_{0}(t)=-\dot{\hat{\vartheta}}_{0}(t) \quad \dot{\tilde{\vartheta}}_{1}(t)=-\dot{\hat{\vartheta}}_{1}(t) \\
& \dot{\tilde{\vartheta}}_{2}(t)=-\dot{\hat{\vartheta}}_{2}(t)
\end{aligned}
$$

has been utilized. After cancelling common terms and then utilizing (43)-(46) and Property 4, we obtain the following expression (see Appendix $\mathrm{C}$ for explicit details)

$$
\dot{V}_{1} \leq-k_{1} e_{1}^{2}-k_{3} e_{3}^{2}-k_{\eta 1} \eta_{1}^{2}-k_{\eta 2} \eta_{2}^{2} .
$$

Hence, utilizing (51) and (54), we can conclude that $e(t), \eta(t)$, $\tilde{\vartheta}_{0}(t), \tilde{\vartheta}_{1}(t), \tilde{\vartheta}_{2}(t) \in \mathcal{L}_{\infty}$ and that $e_{1}(t), e_{3}(t), \eta(t) \in \mathcal{L}_{2}$. Since $e(t), \eta(t), \tilde{\vartheta}_{0}(t), \tilde{\vartheta}_{1}(t), \tilde{\vartheta}_{2}(t) \in \mathcal{L}_{\infty}$, we can utilize (43)-(46) and (48) to conclude that $\hat{\vartheta}_{0}(t), \hat{\vartheta}_{1}(t), \hat{\vartheta}_{2}(t), \dot{\hat{\vartheta}}_{0}(t)$, $\dot{\hat{\vartheta}}_{1}(t), \dot{\hat{\vartheta}}_{2}(t), \Omega_{1}(t), \Omega_{2}(t) \in \mathcal{L}_{\infty}$. Furthermore, from the fact that $e(t), \eta(t), \hat{\vartheta}_{0}(t), \hat{\vartheta}_{1}(t), \hat{\vartheta}_{2}(t) \in \mathcal{L}_{\infty}$, we can utilize Property 4 (i.e., $y_{1} \hat{\vartheta}_{1}, y_{2} \hat{\vartheta}_{2}>0$ ) along with (27), (28), (41), (42), and Appendix A to conclude that $\bar{u}(t), \bar{u}_{d}(t), \tau(t), \tau_{0}(t) \in \mathcal{L}_{\infty}$. Since $e(t), \bar{u}(t) \in \mathcal{L}_{\infty}$, we can utilize (21), (24), and (25) to obtain the fact that $\bar{v}(t), \bar{q}(t) \in \mathcal{L}_{\infty}$; hence, from (18) and (19), it is straightforward to show that $v(t), q(t) \in \mathcal{L}_{\infty}$. From the fact that $e(t), \eta(t), \bar{v}(t), \tilde{\vartheta}_{0}, \tilde{\vartheta}_{1}, \tilde{\vartheta}_{2} \in \mathcal{L}_{\infty}$ and that $y_{1} \hat{\vartheta}_{1}, y_{2} \hat{\vartheta}_{2}>0$, we can conclude that $\dot{e}(t), \dot{\eta}(t) \in \mathcal{L}_{\infty}$ (which is a sufficient condition for $e(t)$ and $\eta(t)$ to be uniformly continuous). Based on the boundedness of the aforementioned signals, we can take the time derivative of $\dot{e}_{3}(t)$ and show that $\ddot{e}_{3}(t) \in \mathcal{L}_{\infty}$ (see 
Appendix B for explicit details). Standard signal chasing arguments can now be used to show that all remaining signals are bounded.

From the fact that $e_{1}(t), e_{3}(t), \eta(t) \in \mathcal{L}_{2}$ and are all uniformly continuous, we can now employ a corollary to Barbalat's Lemma [22] to conclude that

$$
\lim _{t \rightarrow \infty} e_{1}(t), e_{3}(t), \eta(t)=0 .
$$

Next, since $\ddot{e}_{3}(t) \in \mathcal{L}_{\infty}$, we know that $\dot{e}_{3}(t)$ is uniformly continuous. Since we know that $\lim _{t \rightarrow \infty} e_{3}(t)=0$, and $\dot{e}_{3}(t)$ is uniformly continuous, we can use the following equality:

$$
\lim _{t \rightarrow \infty} \int_{0}^{t} \frac{d}{d \tau}\left(e_{3}(\tau)\right) d \tau=\lim _{t \rightarrow \infty} e_{3}(t)+\gamma_{3}
$$

and Barbalat's Lemma [22] to conclude that $\lim _{t \rightarrow \infty} \dot{e}_{3}(t)=0$ where $\gamma_{3} \in \Re^{1}$ is a constant of integration. Based on the fact that $\lim _{t \rightarrow \infty} e_{3}(t), \dot{e}_{3}(t), \eta_{2}(t)=0$, it is straightforward from the expression for $\dot{e}_{3}(t)$ given in (29) to see that

$$
\lim _{t \rightarrow \infty} \frac{\bar{v}_{r 1}(t) \sin \left(e_{3}(t)\right) e_{2}(t)}{e_{3}(t)}=0 .
$$

From (50), and the fact that

$$
\lim _{e_{3} \rightarrow 0} \frac{\sin \left(e_{3}\right)}{e_{3}}=1
$$

we can now conclude that $\lim _{t \rightarrow \infty} e_{2}(t)=0$. The global asymptotic result given in (49) can now be directly obtained from the inverse of the relationship given in (21). That is, from

$$
\left[\begin{array}{l}
\tilde{x} \\
\tilde{y} \\
\tilde{\theta}
\end{array}\right]=\left[\begin{array}{ccc}
\cos \bar{\theta} & -\sin \bar{\theta} & 0 \\
\sin \bar{\theta} & \cos \bar{\theta} & 0 \\
0 & 0 & 1
\end{array}\right]\left[\begin{array}{l}
e_{1} \\
e_{2} \\
e_{3}
\end{array}\right]
$$

it is clear that since $\lim _{t \rightarrow \infty} e_{1}(t), e_{2}(t), e_{3}(t)=0$ that $\lim _{t \rightarrow \infty} \tilde{x}(t), \tilde{y}(t), \tilde{\theta}(t)=0$.

Remark 2: A physical interpretation of the reference trajectory restriction given in (50) is that the reference linear velocity of the WMR must be non zero in the limit, and hence, the WMR regulation problem is not solved as a special case of the adaptive tracking controller (i.e., the WMR cannot be allowed to stop indefinitely at a desired position and orientation). This is a common problem endemic to many tracking controllers presented in literature; however, we have recently developed controllers that overcome this restriction (see [4]) for WMRs that do not rely on visual-servoing. Future research will leverage off of our recent results (see [4]) to address the problem of eliminating the reference trajectory restriction given in (50) for the visual-servoing problem.

\section{SiMULATION AND EXPERIMENTAL IMPLEMENTATION}

In the following section, we provide simulation and experimental results to demonstrate the performance of the adaptive tracking controller given by (28) and (41)-(46). Due to some limitations in the experimental testbed, we believe that the experimental results do not adequately illustrate the performance of the controller; hence, we elected to include simulation results to illustrate the theoretical validity of the proposed controller.

The proposed adaptive tracking controller was simulated and experimentally implemented based on the camera model given in (6)-(9) as follows:

$$
\begin{aligned}
& {\left[\begin{array}{l}
\bar{x}_{c}(t) \\
\bar{y}_{c}(t)
\end{array}\right]=\left[\begin{array}{cc}
\alpha_{1} & 0 \\
0 & \alpha_{2}
\end{array}\right]\left[\begin{array}{cc}
\cos \left(\theta_{0}\right) & -\sin \left(\theta_{0}\right) \\
\sin \left(\theta_{0}\right) & \cos \left(\theta_{0}\right)
\end{array}\right]} \\
& \cdot\left(\left[\begin{array}{l}
x_{c}(t) \\
y_{c}(t)
\end{array}\right]-\left[\begin{array}{l}
O_{o 1} \\
O_{o 2}
\end{array}\right]\right)+\left[\begin{array}{l}
O_{i 1} \\
O_{i 2}
\end{array}\right]
\end{aligned}
$$

and the dynamic model for a modified Pioneer II WMR manufactured by ActivMedia given as follows:

$$
\begin{aligned}
\frac{1}{r_{o}}\left[\begin{array}{cc}
1 & 0 \\
0 & \frac{L_{o}}{2}
\end{array}\right]\left[\begin{array}{l}
\tau_{1} \\
\tau_{2}
\end{array}\right]= & {\left[\begin{array}{cc}
m_{o} & 0 \\
0 & I_{o}
\end{array}\right]\left[\begin{array}{l}
\dot{v}_{1} \\
\dot{v}_{2}
\end{array}\right] } \\
& +\left[\begin{array}{cc}
F_{s 1} & 0 \\
0 & F_{s 2}
\end{array}\right]\left[\begin{array}{l}
\operatorname{sgn}\left(v_{1}\right) \\
\operatorname{sgn}\left(v_{2}\right)
\end{array}\right]
\end{aligned}
$$

where $\alpha_{1}=1$ (pixel $/ \mathrm{m}$ ) and $\alpha_{2}=1$ (pixel $/ \mathrm{m}$ ) represent camera parameters originally defined in $(8), \theta_{0}=0.5(\mathrm{rad})$ represents the camera orientation originally defined in (9), $r_{o}=0.0825$ (m) denotes the radius of the wheels, $L_{o}=0.1635$ (m) denotes the length of the axis between the wheels, $m_{o}=24.8(\mathrm{~kg}) \mathrm{de}-$ notes the mass of the robot, and $I_{o}=0.9453\left(\mathrm{~kg} \cdot \mathrm{m}^{2}\right)$ denotes the inertia of the robot, and $F_{s 1}=1(\mathrm{Nm})$ and $F_{s 2}=1(\mathrm{Nm})$ denote static friction coefficients. The parameter values given above were required to simulate the proposed controller. The parameter values for $r_{o}, L_{o}, m_{o}$, and $I_{o}$ were selected based on approximate measurements or calculations made from the experimental testbed, while parameter values for $\alpha_{1}, \alpha_{2}, \theta_{0}, F_{s 1}$, and $F_{s 2}$ were selected for simplicity. To experimentally verify the proposed adaptive tracking controller we only require knowledge of the torque transmission parameters given by $r_{o}$ and $L_{o}$, due to the fact that the controller is constructed to adapt for uncertainty in the remaining camera and WMR parameters.

\section{A. Simulation Results}

A path planning algorithm [4] was utilized to generate the sinusoidal camera-space reference trajectory illustrated in Fig. 2. The output of this path planning algorithm were the reference camera-space velocity signals $\bar{v}_{r 1}(t)$ and $\bar{v}_{r 2}(t)$ given as follows:

$$
\begin{aligned}
& \bar{v}_{r 1}=4(\text { pixels } / \mathrm{s}) \\
& \bar{v}_{r 2}=\frac{\left.-2.5 \sin \left(0.25 \bar{x}_{c r}\right) \cos \left(\bar{\theta}_{r}\right)\right)}{1+\tan ^{2} \bar{\theta}_{r}}(\mathrm{rad} / \mathrm{s})
\end{aligned}
$$

where the initial conditions for the reference Cartesian cameraspace positions and orientation were selected as follows:

$$
\begin{aligned}
\bar{x}_{c r}(0) & =0 \text { (pixels), } \quad \bar{y}_{c r}(0)=0 \text { (pixels) } \\
\bar{\theta}_{r}(0) & =1.19(\mathrm{rad}) .
\end{aligned}
$$

The initial conditions for the actual Cartesian camera-space positions and orientation were selected as follows:

$$
\begin{aligned}
\bar{x}_{c}(0) & =2.0(\text { pixels }), \quad \bar{y}_{c}(0)=2.0(\text { pixels }) \\
\bar{\theta}(0) & =0.5(\mathrm{rad})
\end{aligned}
$$




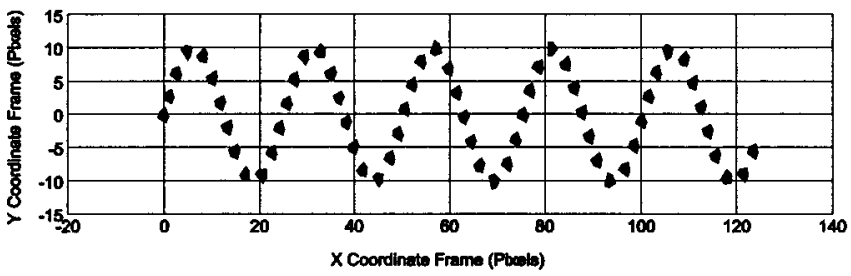

Fig. 2. Desired trajectory.
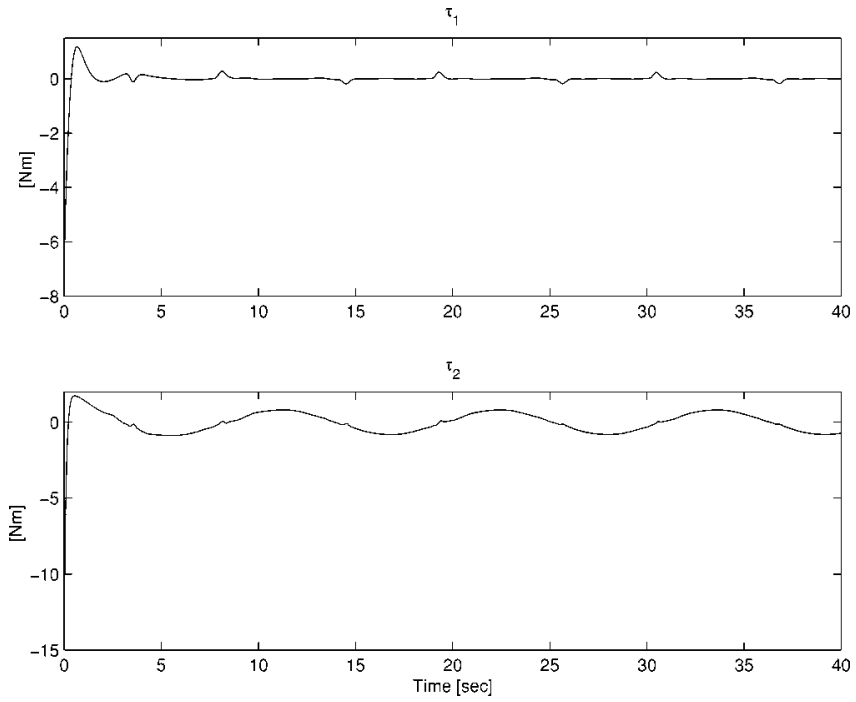

Fig. 3. Camera-space position and orientation tracking errors.

and the initial task-space orientation was selected as follows:

$$
\theta(0)=0
$$

The control gains were tuned until the best response was obtained and then recorded as follows:

$$
\begin{aligned}
k_{1}= & 1.0, \quad k_{3}=2.0 \quad k_{\eta 1}=20.0, \quad k_{\eta 2}=20.0 \\
\Gamma_{0}= & \operatorname{diag}\{7,7,7,10,10,10,10,5,20,5,15, \\
& 7,10,10,10,5,5,5,15,5,15,1,25,5\} \\
\Gamma_{1}= & \operatorname{diag}\{2,2,2,2\} \\
\Gamma_{2}= & \operatorname{diag}\{5,5,5,5,5,5\}
\end{aligned}
$$

where each element of the estimate vector $\hat{\vartheta}_{0}(t)$ was initialized to 0.0 , and each element of the estimate vectors $\hat{y}_{1}(t)$, $\hat{\vartheta}_{2}(t)$ were initialized to 1.0 to ensure $\hat{\vartheta}_{1}(0) \in \operatorname{int}\left(\Lambda_{1}\right), \hat{\vartheta}_{2}(0)$ $\epsilon \operatorname{int}\left(\Lambda_{2}\right)$ (see Property 4 and the discussions in [2] and [12] for further details). The camera-space position and orientation tracking errors are shown in Fig. 3 and the associated control torque inputs are shown in Fig. 4.

\section{B. Experimental Configuration}

To illustrate the real-time performance of the proposed adaptive tracking controller given in (28) and (42)-(46), an experimental testbed (see Fig. 5) was constructed consisting of the following components:

1) modified Pioneer II WMR manufactured by ActivMedia, Inc.;
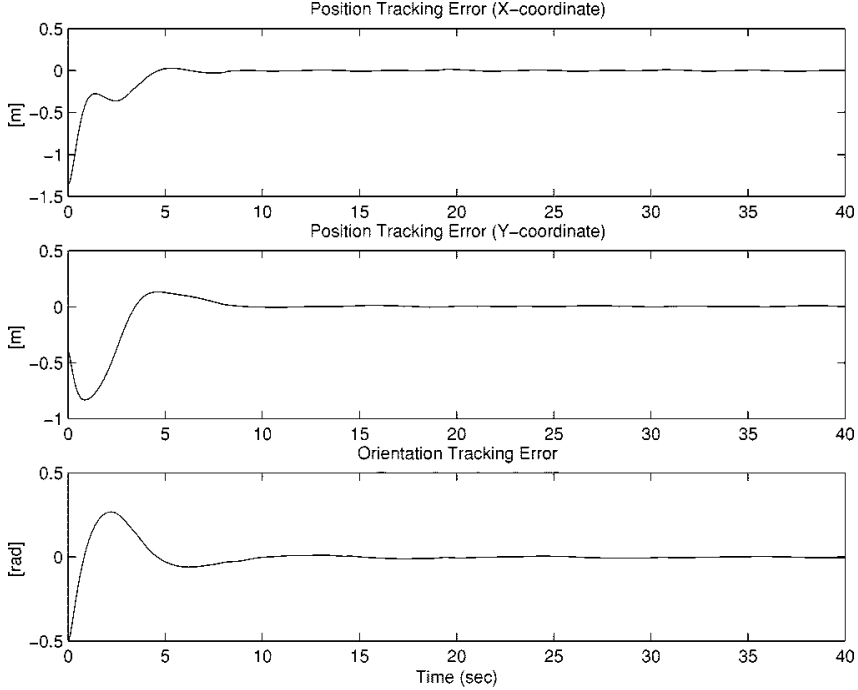

Fig. 4. Control torque inputs.

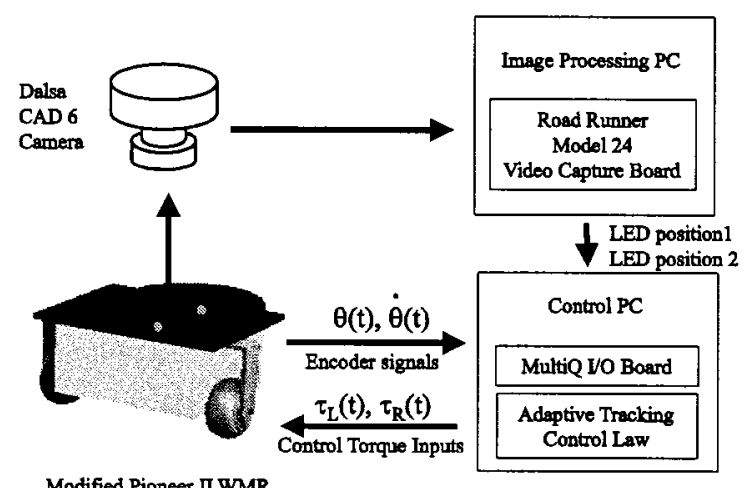

Fig. 5. Experimental testbed.

2) Dalsa CAD-6 camera that captures 955 frames/s with 8 -bit gray scale at a $260 \times 260$ resolution;

3) Road Runner Model 24 video capture board;

4) two Pentium II-based personal computers (PCs) operating under the real-time operating system QNX.

The WMR modifications include

1) replacement of all the existing computational hardware/software with an off-board Pentium $133 \mathrm{MHz}$ PC;

2) replacement of the pulse-width modulated amplifiers and power transmission circuitry with linear amplifiers and the associated circuitry;

3) inclusion of two LEDs (with distinct brightness values) mounted on the top plate of the WMR (one LED was mounted at the COM and the other LED was mounted at the front of the WMR).

The camera was equipped with a $6 \mathrm{~mm}$ lens and mounted 2.87 $\mathrm{m}$ above the robot workspace. The camera was connected to the image processing PC to capture images of the WMR via the video capture board and then determine the positions of the LEDs in the camera-space. The positions of the LEDs were calculated using a threshold based approach that compares brightness values of pixels within a specific range (the brightness of each LED was adjusted to yield a specific signature so that we could distinguish each LED) and selects the brightest pixel in 
the two ranges to be the actual locations of the LEDs in the camera-space. The image processing $\mathrm{PC}$ was connected to a second off-board PC via a dedicated $100 \mathrm{Mb} / \mathrm{s}$ network connection. The second off-board PC was utilized to

1) determine the position, orientation, and linear and angular velocity of the WMR in the camera-space from the LED positions;

2) acquire the task-space orientation of the WMR;

3) execute the control algorithm.

Since an LED was placed above the COM of the WMR, the camera-space position of the WMR was directly given. The camera-space orientation of the WMR was calculated using simple geometric principles that relate the relative position of the two LEDs. The time derivative of the camera-space position and orientation was calculated via a standard backward difference/filtering algorithm while the linear and angular velocities were calculated from (5). In order to determine the task-space orientation of the WMR, we first measured the rotor position of the wheel motors via encoders with a resolution of $0.176^{\circ} /$ line (i.e., 2048 lines/rev). Based on the position of the left and right wheels, denoted by $\theta_{L}(t)$ and $\theta_{R}(t)$, respectively, we obtained the orientation of the WMR through the following static relationship:

$$
\theta=\frac{r}{2 R}\left(\theta_{L}-\theta_{R}\right)
$$

where $r \in \Re^{1}$ denotes the known radius of the wheels, and $R \in \Re^{1}$ denotes the known distance between the wheels. The data acquisition and control execution was performed at $700 \mathrm{~Hz}$ via the Quanser MultiQ Server Board and in-house designed interfacing circuitry. The control algorithms were implemented in $\mathrm{C}++$ and executed using the real-time control environment Qmotor 3.0 [19]. The computed torques were applied to permanent magnet dc motors attached to the left and right wheels via a 19:1 gear coupling. For simplicity, the electrical dynamics of the system were ignored. That is, we assume that the computed torque is statically related to the voltage input of the permanent magnet dc motors by a constant.

\section{Experimental Results}

In order to limit the workspace to a reasonable size for the camera system, we selected the reference linear and angular velocities as follows:

$$
\begin{aligned}
& v_{r 1}=48(1-\exp (-0.05 t))(\text { pixels } / \mathrm{s}) \\
& v_{r 2}=0.64(1-\exp (-0.05 t))(\mathrm{rad} / \mathrm{s})
\end{aligned}
$$

while reference camera-space position/orientation was initialized as follows:

$$
\begin{aligned}
\bar{x}_{c r}(0) & =132 \text { (pixels), } \quad \bar{y}_{c r}(0)=36 \text { (pixels) } \\
\bar{\theta}_{r}(0) & =0.032(\mathrm{rad})
\end{aligned}
$$

and the task-space orientation was initialized as follows:

$$
\theta(0)=0(\mathrm{rad})
$$

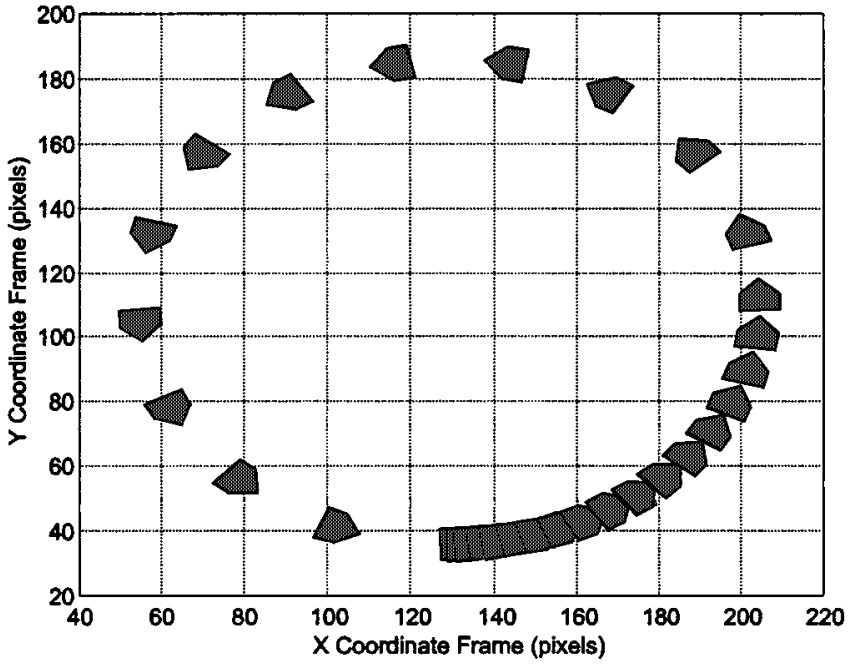

Fig. 6. Desired camera-space trajectory.

Note that the task-space position is unknown. The resulting camera-space reference trajectory is given in Fig. 6. Note that the "soft start" nature of the reference linear and angular velocities is illustrated in Fig. 6 by the arrangement of the polygons which represent the camera-space WMR. The control gains were tuned until the best response was obtained and then recorded as follows:

$$
\begin{aligned}
k_{1}= & 5.5, \quad k_{3}=500 \quad k_{\eta 1}=25.0, \quad k_{\eta 2}=5.5 \\
\Gamma_{0}= & \operatorname{diag}\{0.025,0.0001,0.00005,0.001,0.00005 \\
& 0.00005,0.00005,0.005,0.00001,0.0001,0.0005 \\
& 0.0001,0.0005,0.0001,0.0001,0.00005,0.0001 \\
& 0.0002,0.00005,0.0001,0.00005,0.0001 \\
& 0.0001,0.00002\} \\
\Gamma_{1}= & \operatorname{diag}\{0.0001,0.0001,0.0001,0.002\}
\end{aligned}
$$$$
\Gamma_{2}=\operatorname{diag}\{0.0001,0.00001,0.0001,0.0001,0.00001,0.0001\}
$$

where each element of the estimate vector $\hat{\vartheta}_{0}(t)$ was initialized to 0.0 , and each element of the estimate vectors $\hat{\vartheta}_{1}(t), \hat{\vartheta}_{2}(t)$ were initialized to 15.0 and 25.0, respectively, to ensure $\hat{\vartheta}_{1}(0)$ $\in \operatorname{int}\left(\Lambda_{1}\right), \hat{\vartheta}_{2}(0) \in \operatorname{int}\left(\Lambda_{2}\right)$. The camera-space position and orientation tracking errors are shown in Fig. 7 and the associated control torque inputs are shown in Fig. 8. Note the control torque inputs plotted in Fig. 8 represent the torques applied after the gearing mechanism.

\section{Discussion of Experimental Results}

From the experimental results illustrated in Fig. 7, we can conclude that the proposed adaptive controller achieves reasonable position tracking; however, the orientation tracking performance may not be suitable for many applications. Based on our experience with the experimental hardware, we judge that the lack of orientation tracking performance is due to limitations in the experimental testbed rather than the controller design. One of the obstacles in implementing the proposed controller was determining the position and orientation of the WMR in the camera-space in a simple, efficient manner. To 

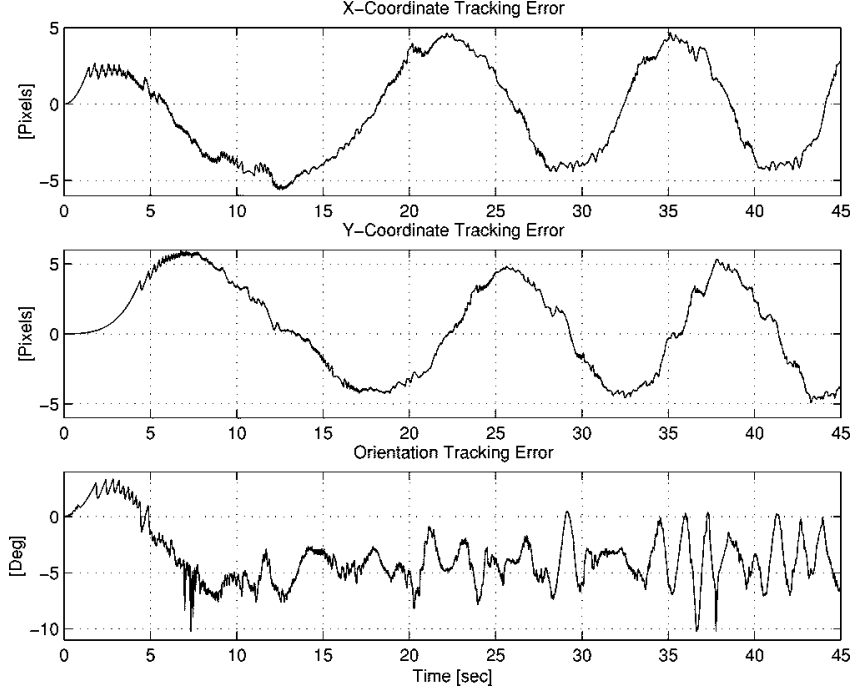

Fig. 7. Position and orientation tracking errors.
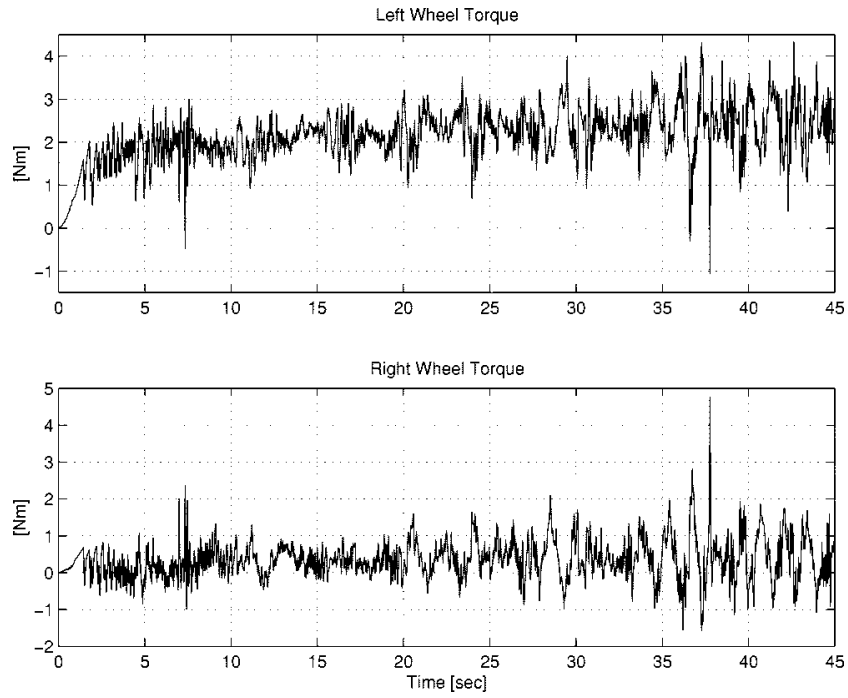

Fig. 8. Control torque inputs.

address this obstacle, we elected to use a threshold algorithm to find the position of the LEDs in the camera-space. That is, each LED appeared as a ring of brightness values (since each LED had a specific brightness signature, two separate rings were clearly distinguishable) and the brightest pixel in the region was selected as the actual location of the LED in the camera-space. Unfortunately, as the WMR moved in the workspace, the LEDs may have been positioned so that the brightest pixel in the image did not correspond to the actual LED location. In addition, if several pixels have the same brightness value, the first pixel location that had the highest brightness value was latched and subsequent pixels with the same brightness value would be neglected. Hence, it is clear that the lack of a more sophisticated, high-speed, image processing algorithm resulted in degraded and noisy LED position measurements. Since the position of the WMR only required the measurement of the position of one LED, the proposed controller was able to achieve reasonable performance. Unfortunately, the measurement of the position of two LEDs is required to determine the orientation of the WMR. Since both LED positions were subject to error and noise, the resulting calculation for the orientation of the WMR was greatly compromised. In conclusion, we believe that if the aforementioned measurement obstacles could be eliminated through a more sophisticated image processing algorithm, the position/orientation tracking error given in Fig. 7 could be decreased further.

\section{CONCLUSIONS}

In this paper, we have presented a global asymptotic position/orientation tracking controller for a WMR that adapts for parametric uncertainty associated with the camera calibration (e.g., magnification factors, focal length, and initial orientation) in addition to the uncertainty associated with the mechanical parameters of the WMR dynamic model (e.g., mass, inertia, friction). The torque input adaptive controller achieves global asymptotic tracking and eliminates the need for integrating the nonlinear kinematic model to obtain the WMR Cartesian position for use in the closed-loop control strategy; hence, we believe the vision-based control approach for WMRs holds the potential for higher performance. In addition, since the trajectory generator is calculated in the camera-space, the proposed approach has the potential for incorporating other desirable features such as avoiding moving objects. Experimental and simulation results were presented to illustrate the performance of the proposed adaptive controller.

\section{APPENDIX A}

In order to show that $T_{1}(\cdot)>\gamma_{1}$, we utilize (10) to conclude that

$$
\bar{v}_{1}=\sqrt{\dot{\bar{x}}_{c}^{2}+\dot{\bar{y}}_{c}^{2}}
$$

where we have used the assumption that the camera system can distinguish between a forward and a reverse motion of the WMR. Upon utilization of (10), we can now formulate a relationship between the actual linear velocity of the WMR and the camera-space linear velocity of the WMR by rewriting (77) as follows:

$$
\bar{v}_{1}=v_{1} \sqrt{\alpha_{1}^{2} \cos ^{2}\left(\theta+\theta_{0}\right)+\alpha_{2}^{2} \sin ^{2}\left(\theta+\theta_{0}\right)} .
$$

From (5) and (10), we can obtain the following expressions for $\dot{\bar{x}}_{c}(t)$

$$
\dot{\bar{x}}_{c}=v_{1} \alpha_{1} \cos \left(\theta+\theta_{0}\right)=\bar{v}_{1} \cos (\bar{\theta}) .
$$

Hence, after substituting (78) for $\bar{v}_{1}(t)$ in (79), we can solve for $\cos (\bar{\theta}(t))$ as follows:

$$
\cos (\bar{\theta})=\frac{\alpha_{1} \cos \left(\theta+\theta_{0}\right)}{\sqrt{\alpha_{1}^{2} \cos ^{2}\left(\theta+\theta_{0}\right)+\alpha_{2}^{2} \sin ^{2}\left(\theta+\theta_{0}\right)}} .
$$

By utilizing similar arguments, we can obtain the following expression for $\sin (\bar{\theta}(t))$ :

$$
\sin (\bar{\theta})=\frac{\alpha_{2} \sin \left(\theta+\theta_{0}\right)}{\sqrt{\alpha_{1}^{2} \cos ^{2}\left(\theta+\theta_{0}\right)+\alpha_{2}^{2} \sin ^{2}\left(\theta+\theta_{0}\right)}} .
$$


Finally, we can substitute (80) and (81) into (13) to conclude that

$$
T_{1}=\frac{1}{\sqrt{\alpha_{1}^{2} \cos ^{2}\left(\theta+\theta_{0}\right)+\alpha_{2}^{2} \sin ^{2}\left(\theta+\theta_{0}\right)}}>\gamma_{1}
$$

where $\alpha_{1}$ and $\alpha_{2}$ are positive scaling factors defined in (8).

\section{APPENDIX B}

In order to show that $\ddot{e}_{3}(t) \in \mathcal{L}_{\infty}$, we take the time derivative of $\dot{e}_{3}(t)$ to obtain the following expression:

$$
\begin{aligned}
\ddot{e}_{3}= & -\frac{\left(\dot{\bar{v}}_{r 1} \sin \left(e_{3}\right) e_{2}\right)}{e_{3}}-\frac{\bar{v}_{r 1} \dot{e}_{3}\left(\cos \left(e_{3}\right) e_{3}-\sin \left(e_{3}\right)\right) e_{2}}{e_{3}^{2}} \\
& -\frac{\bar{v}_{r 1} \sin \left(e_{3}\right) \dot{e}_{2}}{e_{3}}-k_{3} \dot{e}_{3}-\dot{\eta}_{2} .
\end{aligned}
$$

Based on the facts that

$$
\begin{aligned}
\lim _{e_{3} \rightarrow 0} \frac{\sin \left(e_{3}\right)}{e_{3}} & =1 \\
\lim _{e_{3} \rightarrow 0} \frac{\left(\cos \left(e_{3}\right) e_{3}-\sin \left(e_{3}\right)\right)}{e_{3}^{2}} & =0
\end{aligned}
$$

and that $\bar{v}_{r 1}(t), \dot{\bar{v}}_{r 1}(t), e_{2}(t), \dot{e}_{2}(t), \dot{e}_{3}(t), \dot{\eta}_{2}(t) \in \mathcal{L}_{\infty}$, it is straightforward that $\ddot{e}_{3}(t) \in \mathcal{L}_{\infty}$.

\section{APPENDIX C}

In order to show that the expression given in (52) reduces to the expression in given in (54), we substitute for the update law given in (43) and cancel common terms to obtain the following expression:

$$
\begin{aligned}
\dot{V}_{1} \leq & -k_{1} e_{1}^{2}-k_{3} e_{3}^{2}-k_{\eta 1} \eta_{1}^{2}-k_{\eta 2} \eta_{2}^{2} \\
& -\left(\left(\frac{\eta_{1} y_{1} \tilde{\vartheta}_{1}}{y_{1} \hat{\vartheta}_{1}}\right)\left(Y_{d 01} \hat{\vartheta}_{0}+k_{\eta 1} \eta_{1}-e_{1}\right)\right. \\
& \left.+\tilde{\vartheta}_{1}^{T} \Gamma_{1}^{-1} \dot{\hat{\vartheta}}_{1}\right)-\left(\left(\frac{\eta_{2} y_{2} \tilde{\vartheta}_{2}}{y_{2} \hat{\vartheta}_{2}}\right)\right. \\
& \left.\cdot\left(Y_{d 02} \hat{\vartheta}_{0}+k_{\eta 2} \eta_{2}-e_{3}\right)+\tilde{\vartheta}_{2}^{T} \Gamma_{2}^{-1} \dot{\hat{\vartheta}}_{2}\right) .
\end{aligned}
$$

Now, if we substitute for the adaptation laws given in (44)-(46), then we must evaluate (85) for each of the three cases given in (44). In addition to showing (52) reduces to the expression given in (54), we will describe how the parameter update laws given in (44)-(46) ensure that if $\hat{\vartheta}_{i}(0) \in \operatorname{int}\left(\Lambda_{i}\right)$ for $i=1,2$ then $\hat{\vartheta}_{i}(t)$ never leaves the region $\Lambda, \forall t \geq 0$.

Case 1: $\hat{\vartheta}_{i}(t) \in \operatorname{int}\left(\Lambda_{i}\right)$

When the estimate for the parameter vectors $\hat{\vartheta}_{i}(t)$ lies in the interior of the convex region $\Lambda_{i}$, described in Property 4, (86) can be expressed as

$$
\begin{aligned}
\dot{V}_{1} \leq & -k_{1} e_{1}^{2}-k_{3} e_{3}^{2}-k_{\eta 1} \eta_{1}^{2}-k_{\eta 2} \eta_{2}^{2} \\
& -\left(\frac{\eta_{1} y_{1} \tilde{\vartheta}_{1}}{y_{1} \hat{\vartheta}_{1}}\right)\left(Y_{d 01} \hat{\vartheta}_{0}+k_{\eta 1} \eta_{1}-e_{1}\right) \\
& +\tilde{\vartheta}_{1}^{T} y_{1}^{T} \eta_{1} \frac{1}{y_{1} \hat{\vartheta}_{1}}\left(Y_{d 01} \hat{\vartheta}_{0}+k_{\eta 1} \eta_{1}-e_{1}\right) \\
& -\left(\frac{\eta_{2} y_{2} \tilde{\vartheta}_{2}}{y_{2} \hat{\vartheta}_{2}}\right)\left(Y_{d 02} \hat{\vartheta}_{0}+k_{\eta 2} \eta_{2}-e_{3}\right) \\
& +\tilde{\vartheta}_{2}^{T} y_{2}^{T} \eta_{2} \frac{1}{y_{2} \hat{\vartheta}_{2}}\left(Y_{d 02} \hat{\vartheta}_{0}+k_{\eta 2} \eta_{2}-e_{3}\right) ;
\end{aligned}
$$

thus, for Case 1, we can conclude that (52) reduces to the expression in given in (54). In addition, the direction in which the estimate $\hat{\vartheta}_{i}(t)$ is updated for Case 1 is irrelevant, since the worse case scenario is that $\hat{\vartheta}_{i}(t)$ will move toward the boundary of the convex region denoted by $\partial\left(\Lambda_{i}\right)$.

Case 2: $\hat{\vartheta}_{i}(t) \in \partial\left(\Lambda_{i}\right)$ and $\Omega_{i}^{T} \hat{\vartheta}_{i}^{\perp} \leq 0$

When the estimate for the parameter vectors $\hat{\vartheta}_{i}(t)$ lies on the boundary of the convex region $\Lambda_{i}$ described in Property 4 and $\Omega_{i}^{T} \hat{\vartheta}_{i}^{\perp} \leq 0$, then (85) can be expressed as (86); thus, for Case 2, we can conclude that (52) reduces to the expression in given in (54). In addition, the vector $\Omega_{i}$ has a zero or nonzero component perpendicular to the boundary $\partial\left(\Lambda_{i}\right)$ at $\hat{\vartheta}_{i}$ that points in the direction toward the $\operatorname{int}\left(\Lambda_{i}\right)$. Geometrically, this means that $\hat{\vartheta}_{i}$ is updated such that it either moves toward the $\operatorname{int}\left(\Lambda_{i}\right)$ or remains on the boundary; hence, $\hat{\vartheta}_{i}(t)$ never leaves $\Lambda_{i}$.

Case 3: $\hat{\vartheta}_{i}(t) \in \partial\left(\Lambda_{i}\right)$ and $\Omega_{i}^{T} \hat{\vartheta}_{i}^{\perp}>0$ When the estimate for the parameter vectors $\hat{\vartheta}_{i}(t)$ lies on the boundary of the convex region $\Lambda_{i}$ described in Property 4 and $\Omega_{i}^{T} \hat{\vartheta}_{i}^{\perp}>0$, then (85) can be expressed as

$$
\begin{aligned}
\dot{V}_{1} \leq & -k_{1} e_{1}^{2}-k_{3} e_{3}^{2}-k_{\eta 1} \eta_{1}^{2}-k_{\eta 2} \eta_{2}^{2} \\
& -\tilde{\vartheta}_{1}^{T} \Gamma_{1}^{-1}\left(-\Omega_{1}+P_{r}^{t}\left(\Omega_{1}\right)\right) \\
& -\tilde{\vartheta}_{2}^{T} \Gamma_{2}^{-1}\left(-\Omega_{2}+P_{r}^{t}\left(\Omega_{2}\right)\right)
\end{aligned}
$$

where (45) and (46) were utilized. Based on (87), we can utilize Property 4 to conclude that

$$
\begin{aligned}
\dot{V}_{1} \leq & -k_{1} e_{1}^{2}-k_{3} e_{3}^{2}-k_{\eta 1} \eta_{1}^{2}-k_{\eta 2} \eta_{2}^{2} \\
& -\tilde{\vartheta}_{1}^{T} \Gamma_{1}^{-1}\left(-\left(P_{r}^{\perp}\left(\Omega_{1}\right)+P_{r}^{t}\left(\Omega_{1}\right)\right)+P_{r}^{t}\left(\Omega_{1}\right)\right) \\
& -\tilde{\vartheta}_{2}^{T} \Gamma_{2}^{-1}\left(-\left(P_{r}^{\perp}\left(\Omega_{2}\right)+P_{r}^{t}\left(\Omega_{2}\right)\right)+P_{r}^{t}\left(\Omega_{2}\right)\right) \\
\leq & -k_{1} e_{1}^{2}-k_{3} e_{3}^{2}-k_{\eta 1} \eta_{1}^{2}-k_{\eta 2} \eta_{2}^{2} \\
& +\tilde{\vartheta}_{1}^{T} \Gamma_{1}^{-1} P_{r}^{\perp}\left(\Omega_{1}\right)+\tilde{\vartheta}_{2}^{T} \Gamma_{2}^{-1} P_{r}^{\perp}\left(\Omega_{2}\right) .
\end{aligned}
$$

Because $\hat{\vartheta}_{i} \in \partial\left(\Lambda_{i}\right)$, and $\vartheta_{i}$ must lie either on the boundary or in the interior of $\Lambda_{i}$, then the convexity of $\Lambda_{i}$ implies that $\tilde{\vartheta}_{i}(t)$ defined in (48) will either point tangent to $\partial\left(\Lambda_{i}\right)$ or toward $\operatorname{int}\left(\Lambda_{i}\right)$ at $\hat{\vartheta}_{i}(t)$. That is, $\hat{\vartheta}_{i}(t)$ will have a component in the direction of $\hat{\vartheta}_{i}^{\perp}(t)$ that is either zero or negative. In addition, since $P_{r}^{\perp}\left(\Omega_{i}\right)$, points away from $\operatorname{int}\left(\Lambda_{i}\right)$, we have that $\tilde{\vartheta}_{i}^{T} \Gamma_{i}^{-1} P_{r}^{\perp}\left(\Omega_{i}\right) \leq 0$; thus, (88) reduces to (54). Furthermore, since $\hat{\vartheta}_{i}(t)=P_{r}^{t}\left(\Omega_{i}\right)$, we are ensured that $\hat{\vartheta}_{i}(t)$ is updated such that it moves tangent to $\partial\left(\Lambda_{i}\right)$; hence, $\hat{\vartheta}_{i}(t)$ never leaves $\Lambda_{i}$. 


\section{REFERENCES}

[1] B. E. Bishop and M. W. Spong, "Adaptive calibration and control of 2D monocular visual servo system," in Proc. IFAC Symp. Robot Control, Nantes, France, 1997, pp. 525-530.

[2] M. M. Bridges et al., "Control of rigid-link, flexible-joint robots: A survey of backstepping approaches," J. Robot. Syst., vol. 12, no. 3, pp. 199-216, 1995.

[3] C. Canudas de Wit et al., "Nonlinear control for mobile robots," in Recent Trends in Mobile Robots, Y. Zheng et al., Eds. New York: World Scientific, 1993.

[4] W. E. Dixon et al., Nonlinear Control of Wheeled Mobile Robots, Lecture Notes in Control and Information Sciences. New York: SpringerVerlag, Jan. 2000.

[5] B. Espiau et al., "A new approach to visual servoing in robotics," IEEE Trans. Robot. Automat., vol. 8, pp. 313-326, June 1992.

[6] G. D. Hager et al., "Robot hand-eye coordination based on stereo vision," IEEE Control Syst. Mag., vol. 15, pp. 30-39, Feb. 1995.

[7] G. D. Hager and S. Hutchinson, Special Section Vision-Based Control Robot Manipulators, IEEE Trans. Robot. Automat., vol. 12, pp. 651-670, Oct. 1996.

[8] J. Nijmeijer and H. Nijmeijer, "Tracking control of mobile robots: A case study in backstepping," Automatica, vol. 33, no. 7, pp. 1393-1399, 1997.

[9] Y. Kanayama et al., "A stable tracking control method for an autonomous mobile robot," in Proc. IEEE Int. Conf. Robot. Automat., 1990, pp. 384-389.

[10] R. Kelly, "Robust asymptotically stable visual servoing of planar robots," IEEE Trans. Robot. Automat., vol. 12, pp. 759-766, Oct. 1996.

[11] R. Kelly et al., "A two-loops direct visual control of direct-drive planar robots with moving target," in Proc. IEEE Int. Conf. Robot. Automat., 1999 , pp. 599-604.

[12] R. Lozano and B. Brogliato, "Adaptive control of robot manipulators with flexible joints," IEEE Trans. Automat. Contr., vol. 37, pp. 174-181, Feb. 1992.

[13] R. K. Lenz and R. Y. Tsai, "Techniques for calibration of the scale factor and image center for high accuracy 3-D machine vision metrology," IEEE Trans. Pattern Anal. Machine Intell., vol. 10, pp. 713-720, Sept. 1988.

[14] F. Lewis et al., Control of Robot Manipulators. New York: MacMillan, 1993.

[15] Y. Ma et al., "Vision guided navigation for a nonholonomic mobile robot," IEEE Trans. Robot. Automat., vol. 15, pp. 521-596, June 1999.

[16] A. Maruyama and M. Fujita, "Robust visual servo control for planar manipulators with eye-in-hand configurations," in Proc. Conf. Decision Contr., San Diego, CA, Dec. 1997, pp. 2551-2552.

[17] R. McCloskey and R. Murray, "Exponential stabilization of driftless nonlinear control systems using homogeneous feedback," IEEE Trans. Automat. Contr., vol. 42, pp. 614-628, May 1997.

[18] B. Nelson and N. Papanikolopoulos, Special Issue of Visual Servoing, IEEE Robot. Automat. Mag., vol. 5, pp. 521-536, Dec. 1998.

[19] N. P. Costescu et al., "QMotor 3.0 - An object oriented system for PC control program implementation and tuning," in Proc. Amer. Contr. Conf., Arlington, VA, June 2001.

[20] C. Samson and K. Ait-Abderrahim, "Mobile robot control, part 1: Feedback control of a nonholonomic wheeled cart in Cartesian space," INRIA, Sophia-Antipolis, France, Tech. Rep. 1990.

[21] C. Samson et al., Robot Control: The Task Function Approach. Oxford, U.K.: Clarendon, 1991.

[22] S. Sastry and M. Bodson, Adaptive Control: Stability, Convergence, and Robustness. Englewood Cliffs, NJ: Prentice-Hall, 1989.

[23] E. Zergeroglu et al., "Vision-based nonlinear tracking controllers with uncertain robot-camera parameters," in Proc. IEEE/ASME Int. Conf. Advanced Intell. Mechatron., Atlanta, GA, Sept. 1999, pp. 854-859.

[24] E. Zergeroglu et al., "Robust visual-servo control of robot manipulators in the presence of uncertainty," in Proc. IEEE Thirty Eighth Conf. Decision Contr. Phoenix, AZ, Dec. 7-10, 1999, pp. 4137-4142.

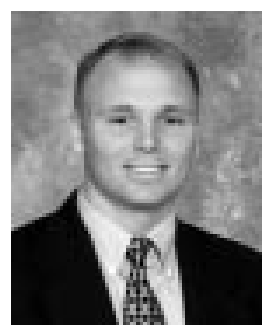

Warren E. Dixon (S'94-M'00) was born in York, PA, in 1972. He received the B.S. and Ph.D. degrees in electrical engineering from Clemson University, Clemson, SC, in 1994 and 2000, respectively, and the M.E. degree in electrical engineering from the University of South Carolina, Columbia, in 1997.

After completing his doctoral studies, he was selected as a Wigner Fellow with the Oak Ridge National Laboratory, Oak Ridge, TN, where he currently works in the Robotics and Process Systems Division. His research interests include mobile robots, fault detection, adaptive and robust control, amplitude limited control, output feedback control, visual-servoing, and the control of underactuated systems.

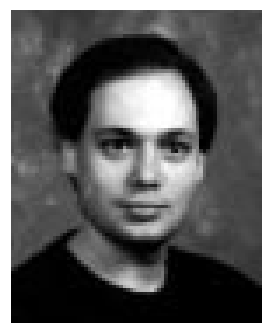

Darren M. Dawson (S'89-M'90-SM'94) was born in Macon, GA, in 1962. He received the Associate degree in mathematics from Macon Junior College in 1982, and the B.S. and Ph.D. degrees in electrical engineering from the Georgia Institute of Technology, Atlanta, in 1984 and 1990, respectively.

From 1985 to 1987, he worked for Westinghouse, Pittsburgh, PA, as a Control Engineer. In July 1990, he joined the Electrical and Computer Engineering Department, Clemson University, Clemson, SC, where he currently is a Professor. He leads the Robotics and Manufacturing Automation Laboratory, which is jointly operated by the Electrical and Mechanical Engineering Departments. His main research interests include nonlinear based robust, adaptive, and learning control with application to electromechanical systems including robot manipulators, motor drives, magnetic bearings, flexible cables, flexible beams, high-speed transport systems, mobile robots, underactuated systems, and aerospace systems.

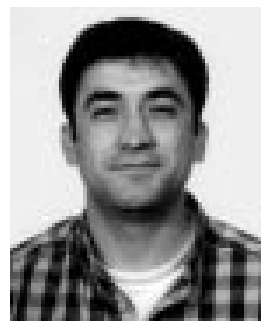

Erkan Zergeroglu (S'98-M'00) was born in Artvin-Yusufeli, Turkey, in 1970. He received the B.S. degree in electrical engineering from Hacettepe University, Ankara, Turkey, in 1992, the M.S. degree from the Middle East Technical University, Ankara, in 1996, and the Ph.D. degree from Clemson University, Clemson, SC, in 2000.

$\mathrm{He}$ is currently with Lucent Technologies, Sturbridge, MA. His research interests include nonlinear control of electromechanical systems with parametric uncertainty, robust and adaptive visual servo control of robotic devices, mobile robotics, model-based control of underactuated mechanical systems, and applied robotics.

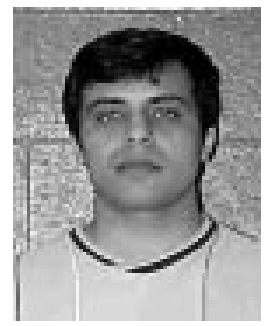

Aman Behal received the M.Tech. degree in electrical engineering from the Indian Institute of Technology, Bombay, in 1996. He is currently pursuing the Ph.D. degree in controls and robotics at Clemson University, Clemson, SC.

His research interests include control of nonlinear systems with special interest in motor control and underactuated systems. 